Review Article

\title{
Neuropathic pain treatment between drug therapy and supplement effects
}

\author{
Mohammed Abdul Muttalib*
}

Department of Therapeutics, Baghdad Collage of Medical Sciences, Baghadad, Iraq

Received: 17 July 2019

Revised: 08 October 2019

Accepted: 09 October 2019

\section{*Correspondence to:}

Dr. Mohammed Abdul Muttalib, Email: mohammed_abdul mutalib@yahoo.com

Copyright: (C) the author(s), publisher and licensee Medip Academy. This is an openaccess article distributed under the terms of the Creative Commons Attribution NonCommercial License, which permits unrestricted noncommercial use, distribution, and reproduction in any medium, provided the original work is properly cited.

\begin{abstract}
Neuropathic pain (NP) is a medical condition induced by diseases or lesions in the primary or inner cell systems that influence somatosensory nervous system buildings. Central NP, including backbone pain; multiple sclerosis, is recurrent. NP has an important impact on the life of patients and a strong economic impact on people and the society. some small neuropathy responds like a NSAID, aspirin and ibuprofen to an over-the-counter drug. More strong medicine (such as anti-depressants and serotonin-epinephrine reuptake inhibitors), anticonvulsants (pregabalin and gabapentin), and topical lidocaine-these are recognized as the most advanced neuropathical treatment-is also needed for other serious circumstances to increase ache leadership. Supplemental drugs, such as beta lipoic acid, acetyl-L-carnitine, benfotiamine, taurine and others, have been studied for neuropathic pain relieve under doctors to guarantee safe use and not bring any medicines that may interact with the dietary supplement. A medical procedure has been investigated for a neuro-lipoic pain relief. In specific, owing to the power of drug contrast with alternative products and owing to the effect of some drugs, it was considered that the drugs are (in spite of their side effects) more helpful and efficient to relieve neuropathic pain than the option, since neuropathic pain represents a serious illness and needs a more strong and effective therapy technology (particularly in severe cases).
\end{abstract}

Keywords: Acetyl-1-carnitine, Anti-depressants, Benfotiamine, Beta lipoic alcohol, Lidocaine, Neuropathic pain, Serotonin-epinephrine reuptake inhibitors

\section{INTRODUCTION}

Neuropathic pain (NP) is a disorder caused by illnesses or lesions influencing somatosensory nervous system structures, either in the main or internal cell systems. ${ }^{1}$

Many prevalent illnesses, accidents and procedures trigger NP by causing lesions in the peripheral or key somatosensory processes. Peripheral NP is the consequence of tiny fiber dysfunction or polyneuropathic diseases (disease, acute alcohol abuse, chemotherapy, amyloidosis, etc. Other triggers include trigeminal neuralgia, herpes zoster, complex regional pain syndrome (CRPS), trapping syndromes, and others. ${ }^{2}$

Central NP is recurrent, including spinal cord injury pain; multiple sclerosis (MS) NP has a significant effect on patient performance of lives and is linked with a heavy financial toll on individuals and community, affecting 8$10 \%$ of the overall workforce. ${ }^{3}$ Neuropathic pain is increasingly recognized as a particular multi-aetiology organization across neuropathic syndromes. ${ }^{4}$

It is increasing incidence due to ageing term population, diabetes mellitus and cancer survival after chemotherapy. 
It is now regarded a separate clinical phenomenon given a wide range of aetiologies. 5

Neuropathy may be split into two main kinds: focal mononeuritis and generalized polyneuropathy symptoms current in many distinct forms, based on the type of nerve cells impacted. The proportion of clients with this form of pain has risen quickly in latest years. According to that neuropathic pain, it impacts about 1 in 10 people and the financial responsibility for the treatment of this pain is rising. ${ }^{6}$ Neuropathic distress impacts about 1 in 10 teenagers and the financial cost for the treatment of this pain is increasing. Research has shown that it is not a pain. Neuropathic suffering improved to $60 \%$ in those with serious clinical neuropathy.

Neuropathic pain is mechanistically unalike with other acute injury situations such as allergic pain that happens, for instance, in rheumatoid arthritis, where the main source is swelling with chemical changes at the injury location; such injury is identified and handled differently. Patients encounter separate symptom characteristics such as tingling and electrical stimuli and pain from nonpainful stimulations (such as light-touching); diseases continue and tend to be permanent and react less to pain medication. Sleep disturbances, serious anxiety and depression in nurses with neuropathic pain, and standard of living is more affected in nurses with acute neuropathic pain than in nurses with acute nonneuropathic pain without harmed or aggravated nerves. ${ }^{8}$

The effective handling of neuropathic pain is evaluated by the degree of comfort and cognitive enhancement encountered by the patient. The handling of neuropathic pain usually compresses feelings because pain can be scarcely handled. Managing aetiological disease like diabetes mellitus is typically inadequate to relieve neuropathic pain. Patients with neuropathic pain usually do not react to analgesics such as acetaminophen, nonsteroidal anti-inflammatory drugs or small opioids as codeine therapy of patients with neuropathic pain is to start therapy with conservative pharmacological and complementary therapies prior to the use of interventional methods such as nerve bends and neuromodulation. ${ }^{6}$

Exercise regimens are techniques that can alleviate neuropathic suffering. However, the connection between workout rate and strength and various metabolic reactions to exercise-induced neuropathic suffering relief remains uncertain. In a research conducted by Sumizono et al, on rats to see the impact of distinct kinds of practice on the decrease of neuropathic pain, the research examined the impact of glial cell activity on neuropathic pain. ${ }^{9}$

\section{PHARMACOLOGICAL DRUG THERAPY}

Antidepressants are one of the earliest medicines used in the therapy of neuropathic distress and have been the topic of many randomized monitored studies and regarded first-line medicines for neuropathic pain. ${ }^{9}$ They were initially used in the therapy of acute distress and, in specific, neuropathic pain because some people suffer from acute pain. The researchers observed that pain relief may be faster in some nurses than antidepressant impact and tends to happen at reduced concentration. The most efficient neuropathic pain antidepressants claim to be tricyclic antidepressant drugs (TCAs). ${ }^{10}$

Tricyclic antidepressant drugs TCAs have been shown to be efficient in several neuropathic circumstances, including traumatic polyneuropathy, post-herpetic neuralgia, superficial nerve disease and severe diabetic neuropathy. On the other side, TCAs's various activities are also liable for many adverse drug reactions such as anticholinergic impacts that were deemed to be the main problem due to the danger of cardiotoxicity, and other adverse effects that include dry mouth, orthostatic hypotension, constipation, and urinary maintenance. To solve these issues, specific serotonin-norepinephrine reuptake inhibitors [SNRIs], especially duloxetine, were implemented to treat neuropathic pain. SNRIs have proved effective under several neuropathic circumstances including severe polyneuropathy, post-herpetic neuralgia, severe diabetic neuropathy, and low back pain. ${ }^{11}$

Strong opioids, such as morphine, oxycodone and hydromorphone, and mild opioids, such as tramadol, have been shown to be used as a second-line treatment of neuropathic pain. This sort of drug is effective when contrasted to other drugs used for neuropathic pain and has comparable effects to antidepressants but has always been regarded as second-line drugs and, more lately, third-line. In general, these drugs should be reserved for patients who cannot accept or struggle to react to firstand second-line drugs. Psychotherapies is another method that has become increasingly common as a pain relief technique. If anxiety increases pain, meditation should assist relieve or decrease pain. ${ }^{12}$ Given their safety, opioid analgesics were recommended second-line medicines; however, they are sometimes used as first option. Third-line treatments include antidepressant drugs (e.g., bupropion, citalopram, and paroxetine) and certain anticonvulsant drugs (e.g., carbamazepine, lamotrigine, oxcarbazepine, and N-methyl-D-aspartate antagonists). These drugs are not fully efficient in neuropathic pain due to the nature of this form of pain and have terrible side-effects and addictive characteristics. In reality, prescription and counter-pain medicines are the fastestgrowing drug addiction and other side effects like sedation, dizziness, edema and ataxia. ${ }^{13}$

Drugs treatment injury or main or peripheral nervous system illness creates some of the most agonizing, intractable acute suffering issues. Pain can lead from immediate trauma (brachial plexus avulsion, phantom limb pain), ischemia (thalamic disease), disease (tabesdorsalis, postherpetic neuralgia), metabolism (diabetic neuropathy) or cancer entry (lumbosacral plexopathy). Patients with these syndromes may portray 
feelings of intolerable heat, searing, squeezing, electrical stimuli, pins and needles, or flesh ripping, often resulting in severe disability. Neuropathic pain pharmacotherapy is difficult and efficient therapy is missing for many clients; suicide is an uncommon but well-described complication of neuropathic pain. ${ }^{14}$

Despite all accessible therapy choices and spinal cord injury (SCI) developments. Medicine, offering appropriate therapy of NP to be challenging. The use of topical lidocaine with TCAs as a localized type of pain management by physicians in the therapy of neuropathic pain. Because it is given straight to the body, only localized joint pain Topical lidocaine occurs in two popular types, a $5 \%$ and an $8 \%$ gel. These two types also block sodium channels in the region of implementation. Mature neurons engaged in pain response depend on sodium channels to propagate pain stimuli, thus totally preventing pain sensation activation. ${ }^{15}$

Although efficient in the therapy of neuropathic pain, opioids are not regarded a first option due to adverse drug reactions and, more lately, due to worries about violence, distraction, and addiction. But in a Japanese research by Sugiyama et al, they saw that Tapentadol is a new drug that has a dual function as both an opioid agonist and noradrenalin reuptake inhibitor. ${ }^{14}$ A proportion of clients with classical pharmacological medication alone do not attain appropriate pain relief. Neurostimulation methods such as transcutaneous electrical nerve stimulation (TENS), central nerve stimuli, organ stem stimulated (NRS), neural cell stimuli (SCS), deep brain stimulation (DBS), epidural muscle lobe stimulated (MCS) and repeated transcranial electric stimuli (rTMS) have shown efficient outcomes in the treatment of acute neuropathic pain. $^{16}$

Research group for studying botanical and synthetic cannabinoids in various pain syndromes (severe distress, cancer pain, chronic non-cancer pain, fibromyalgia pain, migraine, neuropathic pain, visceral pain, and others) found the findings to be blended but cannabinoids generally tend to be most efficient in managing neuropathic pain, allodynia, medication-rebound headache, and chronic nonce. ${ }^{17}$

\section{SUPPLEMENT USED IN THE TREATMENT OF NEUROPATHIC PAIN}

\section{L-acetyl carnitine}

A small molecule of increasing concern in its pharmacological characteristics, is presently sold to treat neuropathic pain. In a controlled randomized trial conducted by Anders and colleagues, ALC has been shown to be effective in decreasing NP and enhancing nerve fiber growth in people with diabetic neuropathy. Acetyl carnitine is a successful compound for the therapy of severe neuropathies because of its dual processes, which include important analgesic impact after acute administration. Moreover, the evident absence of side effects indicates that this drug may be appropriate for long-term treatment and an significant feature of a chronic condition such as neuropathic pain. Thus, up-todate information indicate that L-acetyl carnitine could be used. ${ }^{18}$ Notartomaso and Mascio indicate that 1-acetyl carnitine (LAC) has the distinctive characteristic of longlasting analgesic effects that may decrease relapses in chronic pain clients. ${ }^{19}$

Pract et al researched acetyl-l-carnitine (ALC) in people with human immunodeficiency virus (HIV), disease, and diabetes. Potential intervention processes include adjustment of a defect that may cause neuropathy (sometimes occurring in HIV-positive individuals or those receiving anticonvulsants), a immediate antioxidant impact, or an improved reaction to nerve growth factor. ALC can be administered intramuscularly (IM) or orally in 2000 to $3000 \mathrm{mg} / \mathrm{d}^{20}$

Sheyu and other researchers found that ALC is a basic compound involved in the development of fatty iron in mitochondria and in the regulation of tissue development variables and neurotransmitters in the heart structure Although in latest years efforts have been produced to add ALC to the therapy of diabetic and non-diabetic internal neuropathy, the impacts of ALC under investigated. $^{21}$

Other scholars discovered ALC have acquired clinical awareness in various types of acute pain-related neuropathies, such as diabetes. ALC proved to be efficient in stopping neuropathic pain. Furthermore, ALC is considered to generate a powerful antinociceptive impact after neuropathic pain has been identified. ALC can also enhance peripheral nerve activity by enhancing nerve conductivity, decreasing visual neuronal loss, and encouraging nerve regeneration. Analgesia needs regular ALC administration, indicating the drug controls neuroplasticity across pain neuroaxis. ${ }^{22}$ Vanotti et al, studied ALC, both a symptomatic therapy that can be used in any kind of severe neuropathy, ALC found to have many functions through several processes, causing regeneration of damaged nerve fibres, decreasing oxidative stress, promoting DNA synthesis in mitochondria, and improving nerve growth factor levels in neurons. ${ }^{23}$

\section{Alpha lipoicacid}

Alpha lipoic acid (ALA) is a strong antioxidant that can assist safeguard body cells from harm induced by free radicals' oxidative impacts. Studies indicate that alpha lipoic acid in stopping certain kinds of cell damage within the brain can assist recover vitamin $\mathrm{E}$ and $\mathrm{C}$ concentrations as well as support improve neuron activity and conduction in diabetic nurses. The brain also absorbs it very easily as a complement. Taking alpha lipoic acid as a complement has been shown to assist with body activity and stroke, folly, eye issues, heart disease, 
chronic fatigue syndrome, and diabetes. In the event of neuropathy, surveys have shown that alpha lipoic acid can significantly decrease pain signs such as burning, numbness, itching, or tingling in the hands and ankles connected with peripheral neuropathy frequently found in diabetic patients. ${ }^{24}$ ALA is renowned for its antioxidant characteristics and its function in dehydrogenase responses. ALA is protected against oxidative stress in ischemia-reperfusion lesion, diabetes, neurodegeneration, HIV initiation and others. Recent surveys indicate that ALA performs a much wider function than an antioxidant. $^{25}$

Alpha lipoic acid may be used as an intravenous or capsulated complement. Treatment may require a few decades for people to see complete enhancement with their diseases, but any person who gets from diabetesrelated neuropathy diseases can be life-changing. Alpha lipoic acid has used superficial neuropathy in Germany for years. Additionally, there have been research showing it can assist with another diabetes-related disorder called autonomic neuropathy. Autonomic neuropathy impacts the nerves of internal organs as compared to peripheral neuropathy affecting the nerves of the extremities of the body. $^{24}$

According to the research by Costantino et al, the efficacy of lipoic acid is coupled with a strong safety profile. Data known to date are encouraging and nutritional supplementation with ALA appears to be a helpful instrument for contrasting neuropathic suffering during childbirth. ${ }^{26}$ Mostacci et al suggested that beta lipoic acid was correlated with nausea and swelling in many clients at levels equivalent to or greater than 1200 $\mathrm{mg} / \mathrm{die}^{27}$ The mixture of ALA and superoxide dismutase (SOD) increases efficiency and decreases the use of analgesics in acute high neck discomfort (LBP) by other researchers such as Battisti et al. ${ }^{28}$

Papanas et al, in the pathogenesis-oriented therapy of diabetic neuropathy. ALA's intervention processes in laboratory diabetic neuropathy include reducing oxidative stress along with improving nerve blood flow, nerve conductivity speed, and several other nerve function interventions. ALA is a useful diabetic neuropathy alternative. Compared to previously registered analgesic drugs, it is faster accepted, has a faster initiation of intervention, improving paranaesthesia , numbness, visual deficits, and muscle endurance in relation to neuropathic pain. In clinical exercise, ALA can be selected for nurses with premature neuropathic deficits and diseases where clinical enhancement is more probable. ALA should also be regarded when comorbidities make other analgesics less suitable, or when cardiovascular autonomic neuropathy occurs. ${ }^{29}$

\section{Benfotiamine}

It is a drug extracted from thiamine or vitamin B1. It is thought to raise blood and tissue concentrations and safeguard against extra safety issues due to poor thiamine concentrations. Benfotiamine can be given as a complement, readily discovered in wellness and nutrition shops or normally discovered in garlic, onions, and leeks. This complement has promised to treat neurological and vascular diseases. Additionally, limb disease, sciatica, neuropathy, shingles, fibromyalgia, blood pressure, and coronary disease seem to have positive impacts. While initially used to help diabetic patients, these advantages are accessible to both diabetic and non-diabetic patients. ${ }^{30}$ It was first established in Japan and then used quite frequently in Europe to treat neuropathy. Benfotiamine is Vitamin B1 lipid-soluble. It can normally be discovered in very tiny quantities in fried and broken garlic and other vegetables such as onions, shallots and leeks. However, in order to be efficient as a therapy, the greatest choice is to bring benfotiamine as an oral complement. ${ }^{31}$

According to Nicole benfotiamine, no adverse associations with other drugs have been recognized, and the use of some drugs may effectively trigger a defect in thiamine that can be fixed by using benfotiamine. Most diabetics can predict beneficial outcomes within two to three decades, and will mostly recognize it by seeing improvements in the sore signs they encounter from joint pain. $^{32}$

\section{CONCLUSION}

To pick the most suitable treatment, physicians need a definite concept not only of a patient's amount of pain, but also of the effect that pain has on the patient's standard of lives and other circumstances such as the patient's era, sex other disease the person has, and any prescription the person uses that may contraindicate the treatment.

In particular, due to the potency of drugs comparison with alternatives and that some medications have a placebolike impact, it regarded that the drugs are more helpful and more efficient (despite their side effects) in relieving neuropathic pain than the alternative because neuropathic pain is a serious disorder and (especially in severe instances) need a more powerful and effective therapy technique.

\section{Funding: No funding sources \\ Conflict of interest: None declared \\ Ethical approval: Not required}

\section{REFERENCES}

1. Treede RD, Jensen TS, Campbell JN, Cruccu G, Dostrovsky JO, Griffin JW, et al. Neuropathic pain: redefinition and a grading system for clinical and research purposes. Neurol. 2008;70(18):1630-5.

2. Dworkin RH, Backonja M, Rowbotham MC, Allen RR, Argoff CR, Bennett GJ, et al. Advances in neuropathic pain: diagnosis, mechanisms, and treatment recommendations. Arch Neurol. 2003 Nov $1 ; 60(11): 1524-34$. 
3. Attal N, Fermanian C, Fermanian J, Lantéri-Minet M, Alchaar H, Bouhassira D. Neuropathic pain: are there distinct subtypes depending on the aetiology or anatomical lesion?. Pain. 2008;138(2):343-53.

4. Langley PC, Van Litsenburg C, Cappelleri JC, Carroll D. The burden associated with neuropathic pain in Western Europe. J Medi Econo. 2013 Feb 1;16(1):85-95.

5. Lim EY, Kim YT. Food-derived natural compounds for pain relief in neuropathic pain. Bio Res Intl. 2016.

6. Gaskin DJ, Richard P. The economic costs of pain in the United States. J Pain. 2012 Aug 1;13(8):715-24.

7. Abbott CA, Malik RA, van Ross ER, Kulkarni J, Boulton AJ. Prevalence and characteristics of painful diabetic neuropathy in a large community-based diabetic population in the UK. Diabe care. 2011;34(10):2220-4.

8. Finnerup NB, Haroutounian S, Kamerman P, Baron R, Bennett DL, Bouhassira D, et al. Neuropathic pain: an updated grading system for research and clinical practice. Pain. 2016;157(8):1599.

9. Sumizono M, Sakakima H, Otsuka S, Terashi T, Nakanishi K, Ueda K, et al. The effect of exercise frequency on neuropathic pain and pain-related cellular reactions in the spinal cord and midbrain in a rat sciatic nerve injury model. $J$ pain Res. 2018;11:28.

10. Bayoumi AB, Ikizgul O, Karaali CN, Bozkurt S, Konya D, Toktas ZO. Antidepressants in Spine Surgery: A Systematic Review to Determine Benefits and Risks. Asia spine J. 2019.

11. Lee YC, Chen PP. A review of SSRIs and SNRIs in neuropathic pain. Exp Opin Pharmaco. 2010;11(17):2813-25.

12. Dukefoss TT, Kleggetveit IP, Helås T, Jørum E. Pain and small-fiber affection in hereditary neuropathy with liability to pressure palsies (HNPP). Scan J Pain. 2019 Sep 19.

13. Jensen TS, Madsen CS, Finnerup NB. Pharmacology and treatment of neuropathic pains. Curr Opin Neurol. 2009;22(5):467-74

14. Chabal C, Jacobson L, Mariano A, Chaney E, Britell $\mathrm{CW}$. The use of oral mexiletine for the treatment of pain after peripheral nerve injury. Anesthesiol. 1992;76(4):513-7.

15. Baron R, Binder A, Wasner G. Neuropathic pain: diagnosis, pathophysiological mechanisms, and treatment. Lancet Neurol. 2010;9(8):807-19.

16. Rojewska E, Piotrowska A, Popiolek-Barczyk K, Mika J. Botulinum toxin type A-a modulator of spinal neuron-glia interactions under neuropathic pain conditions. Toxins. 2018;10(4):145.

17. Pergolizzi JJ, LeQuang JA, Taylor JR, Raffa RB, Colucci D. The role of cannabinoids in pain control: the good, the bad, and the ugly. Min Anestesiol. 2018;84(8):955-69.

18. Chiechio S, Copani A, Nicoletti F, Gereau IV. LAcetylcarnitine: A proposed therapeutic agent for painful peripheral neuropathies. Curr Neuropharmacol. 2006;4(3):233-7.

19. Notartomaso S, Mascio G, Bernabucci M, Zappulla C, Scarselli P, Cannella M, et al. Analgesia induced by the epigenetic drug, L-acetylcarnitine, outlasts the end of treatment in mouse models of chronic inflammatory and neuropathic pain. Mol pain. 2017; 13:1744806917697009.

20. Onysko M, Legerski P, Potthoff J, Erlandson M. Targeting neuropathic pain: consider these alternatives. J Fam Pract. 2015;64(8):470-5.

21. Chiechio S, Copani A, Nicoletti F, Gereau IV. LAcetylcarnitine: A proposed therapeutic agent for painful peripheral neuropathies. Curr Neuropharmacol. 2006;4(3):233-7.

22. Chiechio S, Copani A, Gereau RW, Nicoletti F. Acetyl-L-carnitine in neuropathic pain. CNS drugs. 2007;21(1):31-8.

23. Vanotti A, Osio M, Mailland E, Nascimbene C, Capiluppi E, Mariani C. Overview on pathophysiology and newer approaches to treatment of peripheral neuropathies. CNS drugs. 2007;21(1):3-12.

24. Baute V, Zelnik D, Curtis J, Sadeghifar F. Complementary and Alternative Medicine for Painful Peripheral Neuropathy. Currt Treatt Opt Neurol. 2019;21(9):44.

25. Packer L, Witt EH, Tritschler HJ. Alpha-lipoic acid as a biological antioxidant. Free Rad Bio Medi. 1995;19(2):227-50.

26. Costantino M, Guaraldi C, Costantino D, De Grazia S, Unfer V. Peripheral neuropathy in obstetrics: efficacy and safety of a-lipoic acid supplementation. Eur Rev Med Pharmacol Sci. 2014;18(18):2766-71.

27. Mostacci B, Liguori R, Cicero AF. Nutraceutical approach to peripheral neuropathies: evidence from clinical trials. Curr Drug Metab. 2018;19(5):460-8.

28. Battisti E, Albanese A, Guerra L, Argnani L, Giordano N. Alpha lipoic acid and superoxide dismutase in the treatment of chronic low back pain. Eur J Phys Rehabil Med. 2013;49(5):659-4.

29. Papanas N, Ziegler D. Efficacy of $\alpha$-lipoic acid in diabetic neuropathy. Exp Opin Pharmaco. 2014;15(18):2721-31.

30. Nacitarhan C, Minareci E, Sadan G. The effect of benfotiamine on mu-opioid receptor mediated antinociception in experimental diabetes. Exp Clin Endocrinol Diabe. 2014;122(03):173-8.

31. Stracke H, Gaus W, Achenbach U, Federlin K, Bretzel RG. Benfotiamine in diabetic polyneuropathy (BENDIP): results of a randomised, double blind, placebo-controlled clinical study. Exp Clin Endocrinol Diabe. 2008;116(10):600-5.

32. Sánchez-Ramírez GM, Caram-Salas NL, RochaGonzález HI, Vidal-Cantú GC, Medina-Santillán R, Reyes-García G, et al. Benfotiamine relieves inflammatory and neuropathic pain in rats. Eur $\mathbf{J}$ Pharmacol. 2006;530(1-2):48-53.

Cite this article as: Muttalib MA. Neuropathic pain treatment between drug therapy and supplement effects. Int J Basic Clin Pharmacol 2019;8:2573-7. 